\title{
Alpha-mangostin from mangosteen (Garcinia mangostana Linn.) pericarp extract reduces high fat-diet induced hepatic steatosis in rats by regulating mitochondria function and apoptosis
}

Shin-Yu Tsai ${ }^{1}$, Pei-Chin Chung ${ }^{1}$, Eddy E. Owaga ${ }^{2}$, I-Jong Tsai ${ }^{1}$, Pei-Yuan Wang ${ }^{1}$, Jeng-I Tsai ${ }^{3}$, Tien-Shun Yeh ${ }^{4}$ and Rong-Hong Hsieh ${ }^{*}$

\begin{abstract}
Background: Non-alcoholic fatty liver disease (NAFLD) is caused by multiple factors including hepatic oxidative stress, lipotoxicity, and mitochondrial dysfunction. Obesity is among the risk factors for NAFLD alongside type 2 diabetes mellitus and hyperlipidemia. a- mangostin ( $\mathrm{a}-\mathrm{MG}$ ) extracts from the pericarps of mangosteen (Garcinia mangostana Linn.) may regulate high fat diet-induced hepatic steatosis; however the underlying mechanisms remain unknown. The aim of this study was to investigate the regulatory effect of a-MG on high fat diet-induced hepatic steatosis and the underlying mechanisms related to mitochondrial functionality and apoptosis in vivo and in vitro.

Methods: Sprague Dawley (SD) rats were fed on either AIM 93-M control diet, a high-fat diet (HFD), or high-fat diet supplemented with $25 \mathrm{mg} /$ day mangosteen pericarp extract (MGE) for 11 weeks. Thereafter, the following were determined: body weight change, plasma free fatty acids, liver triglyceride content, antioxidant enzymes (superoxide dismutase, SOD; glutathione, GSH; glutathione peroxidase, GPx; glutathione reductase GRd; catalase, CAT) and mitochondrial complex enzyme activities. In the in vitro study, primary liver cells were treated with $1 \mathrm{mM}$ free fatty acid (FFA) (palmitate: oleate acid =2:0.25) to induce steatosis. Thereafter, the effects of a-MG (10 $\mu \mathrm{M}, 20 \mu \mathrm{M}$, $30 \mu \mathrm{M}$ ) on total and mitochondria ROS (tROS, mitoROS), mitochondria bioenergetic functions, and mitochondrial pathway of apoptosis were examined in the FFA-treated primary liver cells.

Results: The MGE group showed significantly decreased plasma free fatty acids and hepatic triglycerides (TG) and thiorbarbituric acid reactive substances (TBARS) levels; increased activities of antioxidant enzymes (SOD, GSH, GPx, GRd, (AT); and enhanced NADH-cytochrome $c$ reductase (NCCR) and succinate-cytochrome $c$ reductase (SCCR) activities in the liver tissue compared with HFD group. In the in vitro study, a-MG significantly increased mitochondrial membrane potential, enhanced cellular oxygen consumption rate (OCR), decreased tROS (total ROS) and mitoROS (mitochondrial ROS) levels; reduced $\mathrm{Ca}^{2+}$ and cytochrome $c$ (cyt c) release from mitochondria, and reduced caspases 9 and 3 activities compared with control group.

(Continued on next page)
\end{abstract}

\footnotetext{
* Correspondence: hsiehrh@tmu.edu.tw

'School of Nutrition and Health Sciences, Taipei Medical University, 250

Wu-Hsing Street, Taipei 110, Taiwan

Full list of author information is available at the end of the article
} 
(Continued from previous page)

Conclusion: These findings demonstrate a-MG attenuated hepatic steatosis in high fat-diet fed rats potentially through enhanced cellular antioxidant capacity and improved mitochondrial functions as well as suppressed apoptosis of hepatocytes. The findings of study represent a novel nutritional approach on the use of a-MG in the prevention and management of NAFLD.

Keywords: a-mangostin, Fat infiltration, Mitochondria dysfunction, Mitochondrial pathway apoptosis, Antioxidant enzymes

\section{Background}

Non-alcoholic fatty liver disease (NAFLD) is among the most common chronic liver diseases and its prevalence is associated with the increase in obesity and other metabolic syndrome conditions [1, 2] The hallmark of NAFLD is hepatic steatosis, which manifests as excessive triglyceride accumulation in the hepatocytes [2]. The pathogenesis of NAFLD is generally described by 'two-hit' theory represented by initial step of hepatic lipid accumulation and thereafter hepatic cellular injury due to lipotoxicity mediated by lipid peroxidation and pro-inflammatory factors [1]. Hepatic steatosis mainly results from disrupted lipid metabolism in the hepatocytes leading to imbalance between intrahepatic triglyceride (TG) accumulation and clearance [3]. Among the underlying reasons for the fat accumulation is the failure of long chain fatty acids catabolism due to impaired hepatic mitochondrial $\beta$-oxidation [1]. The increased FFA flux in the hepatocytes increase the production of free radicals and other reactive oxygen species (ROS) leading to intracellular redox imbalance. Due to the reactive nature of the ROS, the antioxidant enzyme activities are suppressed resulting in oxidative stress and hepatic cellular injury [4].

Hepatic lipid accumulation in NAFLD setting has been associated with mitochondrial dysfunction arising mainly from cellular oxidative damage [1]. Mitochondria are key sources of ROS in the cell mainly due to mitochondrial uncoupling disturbances and inhibition of the respiratory chain leading to free radical leakage in the electron transport chain [5]. A previous study found rat fed on high fat diet had increased levels of free fatty acid, oxidative stress, and decreased antioxidant enzymes activity, which collectively induced mitochondrial proton leakage [6]. The imbalance between the mitochondria antioxidant capacity and generated ROS affects the mitochondria integrity and catabolic functions such as $\beta$-oxidation of fatty acids thereby resulting in excessive hepatic triglyceride accumulation [4].

Apoptosis of hepatocytes is a critical feature of NAFLD and is associated with structural changes in the liver tissues leading to physiological and pathological conditions [7]. The role of mitochondria-dependent apoptosis in the development of hepatic steatosis has been hypothesized by several authors [8]. Oxidative stress can interfere with mitochondria membrane integrity including loss of mitochondrial membrane potential $(\Delta \psi \mathrm{m})$, release of $\mathrm{Ca}^{2+}$, activation of caspases 9 and 3; reduction of $\mathrm{Bcl}-2 / \mathrm{Bax}$ ratio and release of cytochrome $\mathrm{c}$ leading to programmed hepatocytes cell death [9]. The main consequence of hepatocyte apoptosis is dysregulated lipid clearance resulting in triglyceride accumulation. On the other hand, oxidative stress can also cause hepatocytes apoptosis through the redox signalling [8].

In recent years, several studies have explored the modification of the risk factors such as oxidative stress as potential therapeutic targets for NAFLD through the use of antioxidants and dietary bioactive compounds [1, 4]. Mangosteen (Garcinia mangostana Linn.) is a tropical fruit that contains a wide range of bioactive compounds such as xanthones, anthocyanins, tannins and the beneficial health effects have been linked to the anti-oxidative, anti-bacterial, anti-cancer and anti-adipogenic effects $[4,10] . \alpha$ - mangostin ( $\alpha-M G)$ is the main constituent of the fruit hull of the mangosteen, and possesses a wide variety of pharmacological effects including inhibition of inflammation; inhibition of ROS through enhanced anti-oxidative enzymes activities [11]; and improvement of metabolic disorders of high fat-diet fed mice [4]. Previous studies showed $\alpha-M G$ significantly reduced lipid peroxidation and improved antioxidant capacity in fat-infiltrated liver $[4,12]$. Choi et al. found $\alpha-M G$ treatment could alleviate obesity in a mice model through induction of adenosine 5'-monophosphate (AMP)-activated protein kinase (AMPK) protein [12]. Nevertheless, the effect of mangosteen on hepatic lipid homeostasis in NAFLD setting has not been elucidated and the underlying mechanisms related to mitochondria functionality and apoptosis remain unknown. Thus, our study aimed at investigating the regulatory effects of mangosteen pericarp extract on hepatic fat- accumulation in high-fat diet fed rats, and further examine the underlying mechanisms related to mitochondrial functionality and apoptosis in vivo and in vitro.

\section{Methods \\ Reagents}

Palmitic acid, oleic acid, pure $\alpha-$ MG (>99\%), 1\% Penicillin/Streptomycin (PS), and dimethyl sulfoxide (DMSO) were obtained from Sigma-Aldrich (US). Leibovitz's L-15 medium was purchased from ThermoFisher, (US), 
whereas fetal bovine serum (FBS) was obtained from GIBCO Co. (US). Mangosteen pericarp extract was obtained from Shinn Nan World Trade Co., Ltd. (Taipei, Taiwan) and contained 84\% total mangosteen xanthones as $\alpha$-mangostin.

\section{Animal experiment}

The animal experiment protocol was reviewed and approved by the Institutional Animal Care and Use committee (IACUC/IACUP) at Taipei Medical University (IACUC approval No: LAC-2015-0109). Adult male SD rats $(240.2 \pm 1.5 \mathrm{~g}, 6-7$ weeks old were obtained from BioLASCO Taiwan Co., Ltd. (Taiwan) and maintained under specific-free conditions at $22{ }^{\circ} \mathrm{C}$ on a $12 \mathrm{~h}$ lightdark cycle with ad libitum access to food and water. After 1 week of acclimatization, the rats were equally divided into three groups $(n=8)$ : (i)AIN $93 \mathrm{M}$ diet (control) from MP Biomedicals (US) (ii) High fat diet group (HFD) - AIN93M modified high fat diet (iii) MG group (MGE) - $25 \mathrm{mg} /$ day mangosteen extract with AIN93M modified high fat diet. The caloric distribution of the control group was 77\%: 9\%:14\% of energy provided as carbohydrate: fat: protein, respectively whereas the caloric distribution of the HFD group was $28 \%: 60 \%: 11 \%$, of energy provided as carbohydrate: fat: protein, respectively. Lard was used as the major fat source in the diet. The amount of food consumed by the rats in the respective groups were not significantly different i.e. control group - $19.79 \pm 0.34$ g/per day; HFD group- $19.67 \pm$ $0.54 \mathrm{~g} /$ per day; and MGE group- $19.41 \pm 1.02 \mathrm{~g} /$ per day. The rats were weighed every week during the experiment period. After 11 weeks of feeding, the rats were fasted overnight and sacrificed. The liver tissues were removed, weighed and thereafter subjected to histology examination. All samples were stored at $-80{ }^{\circ} \mathrm{C}$ prior to analysis of hepatic triglyceride and free fatty acids content, hepatic oxidative damage expressed as Thiobarbituric acidreactive substances (TBARS), antioxidant enzyme activities, and mitochondrial complex enzyme activities.

\section{Isolation of primary hepatocytes and Free fatty acid (FFA)-treatment experimental design}

Primary hepatocytes were isolated from 6 to 8 weeks old male SD rats weighing 150-200 g, through portal vein collagenase perfusion of liver as per the method of Seglen [13]. Hepatocytes were cultured for $24 \mathrm{~h}$ in Leibovitz's L-15 medium supplemented with $1 \mu \mathrm{mol} / \mathrm{L}$ dexamethasone and $2.5 \%$ FBS (supplemented with $5.6 \mathrm{mM} / \mathrm{L}$ galactose, and $1 \%$ penicillin/streptomycin). A typical yield was about $4-5 \times 10^{7}$ hepatocytes/rats with $>80 \%$ cell viability as determined by trypan blue exclusion assay. In order to induce fat infiltration to simulate hepatic steatosis, rat primary hepatocytes were placed in 6 -well plates $\left(2 \times 10^{5}\right.$ cells $)$ and cultured for $24 \mathrm{~h}$ in $10 \%$
FBS media supplemented with $1 \mathrm{mM}$ free fatty acid (FFA) (palmitate: oleate acid $=2: 1$ ) as previously described [14]. The primary hepatocytes were treated with different $\alpha$-MG concentrations $(10 \mu \mathrm{M}, 20 \mu \mathrm{M}$ and $30 \mu \mathrm{M})$ were dissolved before use DMSO for $24 \mathrm{~h}$. These dosages fall within the ranges previously studied by other authors [15]. Analyses were conducted for cellular oxygen consumption rate (OCR), total and mitochondrial ROS, cytochrome c release, caspase-3 and -9 , and mitochondrial membrane potential.

\section{Analysis of hepatic triglyceride and plasma free fatty acids profile}

The plasma free fatty acid concentrations were determined by enzymatic colorimetric methods using commercial kits (Randox, UK). The lipids were extracted from liver samples using chloroform-methanol method [16]. The liver TG levels in the lipid extracts were determined by enzymatic colorimetric methods using commercial kits (Randox, UK).

\section{Hepatic histology}

Liver tissues were fixed overnight at room temperature in $10 \%$ formaldehyde and embedded in paraffin. Thick sections $(8 \mu \mathrm{m})$ were stained with hematoxylin \& eosin and mounted on glass slides and examined using a microscope (Leica ICC50 HD, Germany) (original magnification $\times 200$ ).

\section{Hepatic TBARS assay}

As a marker of oxidative damage from lipid peroxidation, the TBARS concentrations were measured in the liver tissue and primary hepatocytes using the method of Park et al. [17]. First, the supernatant $(20 \mu \mathrm{L})$ was mixed with $0.22 \% \mathrm{H}_{2} \mathrm{SO}_{4}(800 \mu \mathrm{L})$, phosphotungstic acid (PTA) $(100 \mu \mathrm{L})$ and thiobarbituric acid (TBA) $(200 \mu \mathrm{L})$. The reaction mixture was heated at $95{ }^{\circ} \mathrm{C}$ for $1 \mathrm{~h}$, and cooled for $10 \mathrm{~min}$. Thereafter butanol $(400 \mu \mathrm{L})$ was added to the mixture and vortexed. The mixture was centrifuged at $25{ }^{\circ} \mathrm{C}$ for $15 \mathrm{~min}(650 \times \mathrm{g})$ and the supernatant fluorescence measured using TBARS Assay Kit (Cayman, US) at excitation and emission wavelengths of $530 \mathrm{~nm}$ and $590 \mathrm{~nm}$, respectively.

\section{Assay of hepatic antioxidant enzymes activities}

Liver tissue $(0.5 \mathrm{~g})$ was homogenized in $1.5 \mathrm{~mL}$ buffer (20 M Tris-base,7 VM NaCl, $1 \%$ Triton X-100, pH 7.2, $0.1 \%$ protease inhibitor) and incubated at $4{ }^{\circ} \mathrm{C}$, vortexing every $10 \mathrm{~min}$ up to $90 \mathrm{~min}$. The homogenate was centrifuged at $4{ }^{\circ} \mathrm{C}$, for $15 \mathrm{~min}(3000 \times \mathrm{g})$. The supernatants were collected and stored at $-30{ }^{\circ} \mathrm{C}$ for further analysis. The amount of protein in supernatant was measured using bicinchoninic acid (BCA) kit (ThermoFisher, US). Superoxide dismutase (SOD) activity was analyzed using 
commercial kit (ENZO, UK). The glutathione (GSH) and catalase (CAT) activities were measured using respective commercial kits (Cayman, US). The glutathione reductase (GRd) and glutathione peroxidase (GPx) activities were determined using respective commercial kits (Randox, UK).

\section{Analysis of hepatic mitochondrial complex enzyme activity}

In the determination of nicotinamide adenine dinucleotidecytochrome c reductase (NCCR) activity, $900 \mu \mathrm{L}$ test solution (1 mM NADH, $1.5 \mathrm{mM}$ potassium cyanide, $50 \mathrm{mM}$ potassium phosphate buffer, $\mathrm{pH} 7.4$ ) was added to $200 \mu \mathrm{g}$ of liver mitochondrion extract and incubated at $37{ }^{\circ} \mathrm{C}$ for $3 \mathrm{~min}$. Thereafter, $1 \mathrm{mM}$ oxidized cytochrome $c(100 \mu \mathrm{L})$ was added. The activity absorbance was measured spectrophotometrically at $550 \mathrm{~nm}$ for $3 \mathrm{~min}$. In the succinatecytochrome c reductase (SCCR) activity assay, $900 \mu \mathrm{L}$ test solution (22 mM succinate, $1.66 \mathrm{mM}$ potassium cyanide, $44 \mathrm{mM}$ potassium phosphate buffer, $\mathrm{pH} 7.4$ ), was mixed with liver mitochondrion extract $(200 \mu \mathrm{g})$ and $0.5 \mathrm{mM}$ oxidized cytochrome c $(100 \mu \mathrm{L})$, and incubated at $37{ }^{\circ} \mathrm{C}$ for $2 \mathrm{~min}$. The SCCR activity was measured spectrophotometrically at $550 \mathrm{~nm}$ for $3 \mathrm{~min}$.

Determination of cellular oxygen consumption rate (OCR) The FFA-treated rat primary hepatocyte $\left(5 \times 10^{4}\right.$ cells per well) were cultured in plates for the analysis of the cellular oxygen consumption rate using XFe24 Analyzer plates (Seahorse Bioscience, US). The analyzer was sequentially injected with the three mitochondrial inhibitor compounds (oligomycin, carbonylcyanide p-trifluoromethoxyphenylhydrazone, CCCP; and antimycin A) through the assigned ports. The OCR was expressed as $\mathrm{pMole} / \mathrm{min}$.

Total ROS (tROS) and mitochondrial ROS (mitoROS) assay tROS was determined in the FFA-treated primary hepatocytes by analysis of 2,7-dichlorodihydrofluorescein diacetate $\left(\mathrm{H}_{2}\right.$ DCFDA) using a commercial kit (Cayman, US). mitoROS was examined using MitoSOX ${ }^{\mathrm{s}}$ Red commercial kit (Cayman, US)..

Assay of mitochondrial membrane potential, cytochrome c (cyt c), and caspase ( 3 and 9) activities

Mitochondrial membrane potential was determined using MitoProbe $^{\mathrm{Tm}}$ JC-1 assay kit. (ThermoFisher, US). Cyt $c$ assay was evaluated using cyt $c$ releasing apoptosis assay kit (Biovision, US). The cyt $c$ release was determined by western blotting of the cell homogenate of the cytosolic and mitochondrial fractions. Caspases- 3 and 9 assays were evaluated using caspase-3/CPP32 and 9 colorimetric assay kits, respectively (Biovision, US). The cells were sonicated to obtain protein concentration of $100 \mu \mathrm{g} / \mathrm{mL}$. After addition of reaction buffer and DTT to the protein extract, DEVD-pNA (caspase 3) or LEHD-pNA (caspase 9) substrate were added to the mixture and incubated at $37{ }^{\circ} \mathrm{C}$ for $2 \mathrm{~h}$. The samples absorbance were read at $405 \mathrm{~nm}$ using a spectrophotometer.

\section{Statistical analysis}

Data are expressed as the mean \pm standard deviation. The differences between the mean values were evaluated by ANOVA followed by Duncan's multiple range test. In all analyses, $p$-values $<0.05$ were considered statistically significant.

\section{Results}

Mangosteen pericarps extract markedly reduced body weight gain, plasma free fatty acids (FFA) levels, hepatic triglyceride (TG) accumulation

Mangosteen pericarps extract supplementation significantly decreased body weight in the MGE group compared to HFD group (Fig. 1a). After feeding the SD rats on high fat diet (HFD) for 11 weeks, plasma FFA (Fig. 1b) and hepatic TG concentration (Fig. 1d) were markedly increased in HFD group thereby confirming the primary goal of inducing fat accumulation in the animal model was successful. This is further supported by the hepatic histology images that showed increased lipid deposition in the hepatocytes of the HFD group showed compared to control and MGE groups (Fig. 1c). Mangosteen pericarps extract supplementation significantly reduced hepatic TG concentration (Fig. 1d) and plasma FFA levels (Fig. 1b) MGE group compared to HFD group. Mangosteen pericarps extract also significantly reduced TBARS levels, a marker of lipid peroxidation, in the MGE group (Fig. 1e).

\section{Mangosteen pericarps extract increased hepatic antioxidant enzyme activities and reduced ROS in rat liver tissue}

Mangosteen pericarps extract treatments increased antioxidant enzymes (SOD, GSH, GPx, GRd, CAT) activities in the rat liver tissue in MGE group compared to HFD group (Table 1). These results demonstrate mangosteen extract could confer a protective effect by enhancing hepatic antioxidant enzymes activities. In the in vitro study, $\alpha-M G$ treatment decreased TBARS concentration in FFA-treated hepatocytes (Fig. 2a) and decreased tROS (Fig. 2b) and mitoROS (Fig. 2c) concentration in FFA -treated hepatocytes. Overall, our study found $\alpha-M G$ decreased oxidative stress in FFA -treated hepatocytes.

\section{a-MG suppressed mitochondrial pathway of apoptosis in FFA-treated hepatocytes}

$\alpha$-MG treatments $(10 \mu \mathrm{M}, 20 \mu \mathrm{M}$ and $30 \mu \mathrm{M})$ decreased $\mathrm{Ca}^{2+}$ (Fig. 2e) and cytochrome c levels (Fig. 2d), decreased caspases 9 and 3 activities (Fig.3a), and 

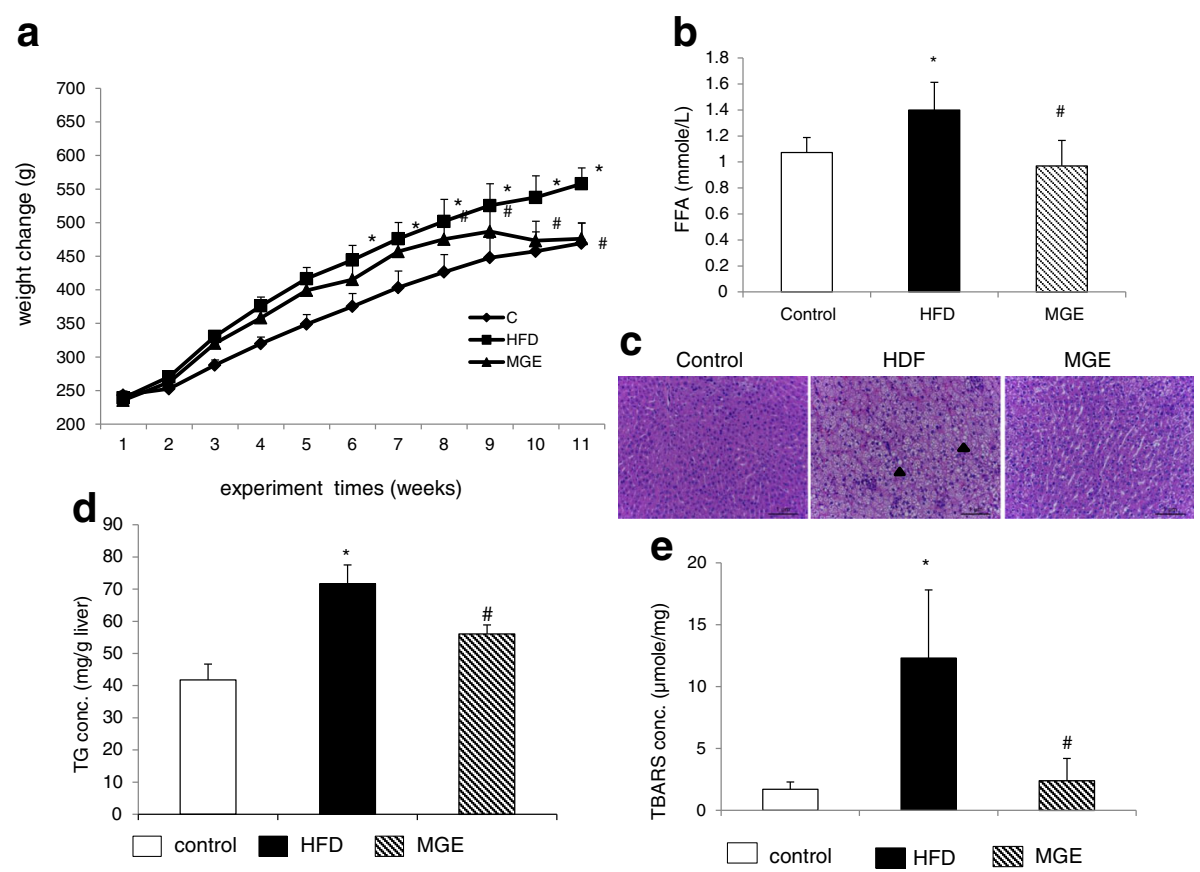

Fig 1 Effect of mangosteen pericarp extracts on: a body weight change, b FFA in plasma, c representative images of hematoxylin and eosin stained liver tissue. Original magnification, ×200, d Liver TG concentration, e Liver TBARS concentration. FFA: free fatty acid, TG: triacylglycerol, TBARS: thiobarbituric acid reactive substances. c: control; HFD: high fat diet; MGE: HFD supplemented with 25 mg/day mangosteen pericarp extract. Values are mean \pm SD $(n=8) ;{ }^{*} P<0.05$ versus control ; ${ }^{*} P<0.05$ versus HFD)

decreased mitochondrial membrane potential (Fig. 3b) in FFA-treated primary hepatocytes. Our results implied $\alpha$-MG suppressed the early phase of mitochondrialdependent apoptosis.

\section{a-MG treatment enhanced mitochondrial function and enzyme activities}

$\alpha$-MG treatment increased NCCR (mitochondrial complex I and III) and SCCR (mitochondrial complex II and III) activities in MGE group compared to HFD group (Fig. 3c). Our results showed significantly increased mitochondrial oxygen consumption rate in the $20 \mu \mathrm{M}$ $\alpha$-MG treatment compared to control in FFA-treated

Table 1 Effect of mangosteen peel extract on hepatic antioxidant enzyme activity in high fat diet-fed rats

\begin{tabular}{llll}
\hline & Control & HFD & MGE \\
\hline GSH (mole/mg protein) & $96.9 \pm 2.2$ & $93.0 \pm 0.7^{*}$ & $96.5 \pm 1.6^{\#}$ \\
GPx (U/mg protein) & $0.98 \pm 0.08$ & $0.85 \pm 0.14^{*}$ & $0.95 \pm 0.08^{\#}$ \\
GRd (U/g protein) & $0.82 \pm 0.2$ & $0.66 \pm 0.14^{*}$ & $0.96 \pm 0.21^{\#}$ \\
SOD (Unit/min/mg protein) & $0.02 \pm 0.03$ & $0.14 \pm 0.05^{*}$ & $0.20 \pm 0.03^{\#}$ \\
CAT (Kunits/mg protein) & $19.5 \pm 2.8$ & $14.3 \pm 5.3^{*}$ & $19.9 \pm 2.6^{\#}$
\end{tabular}

Values are mean $\pm \mathrm{SD}(n=8)$; means without a common letter differ $(p<0.05)$. GSH: glutathione; GPx: glutathione peroxidase; GRd: glutathione reductase; SOD: superoxide dismutase; CAT: catalase. HFD: high fat diet; MGE: HFD supplemented $25 \mathrm{mg} /$ day mangosteen extract diet $\left({ }^{*} P<0.05\right.$ versus control ; $P<0.05$ versus HFD) hepatocytes (Fig. 3d). These data show $\alpha$-MG of mangosteen pericarps extract treatment could improve efficiency of mitochondrial functions such as oxidative phosphorylation and enzyme activities.

\section{Discussion}

We evaluated the regulatory effects of mangosteen pericarp extract on hepatic fat-accumulation in high-fat diet fed rats, and further examine the underlying mechanisms related to mitochondrial functionality and apoptosis in vivo and in vitro. In this study, the weight change in the MGE group was markedly lower than in the HFD-control diet (Fig. 1a), and MGE further suppressed accumulation of free fatty acids (Fig. 1b), lipid deposition in hepatocytes (Fig. 1c) and triglyceride (Fig. 1d) compared to the levels in the HFD group. We report here for the first time, that the MGE suppressed effect on hepatic triglyceride accumulation could be directly or indirectly attributed to modulation of the antioxidant capacity, mitochondrial bioenergetic functionality and mitochondria-dependent apoptosis. Our findings on suppressed triglyceride were in agreement with a previous study that showed improved biochemical serum profiles (total cholesterol, triglyceride, and fatty acid) in high fat diet fed mice after supplementation with $\alpha$-MG $(50 \mathrm{mg} / \mathrm{kg})$ for 80 days [4]. Choi et al. also reported anti-obesity effects of $\alpha-M G$ in HFD induced 


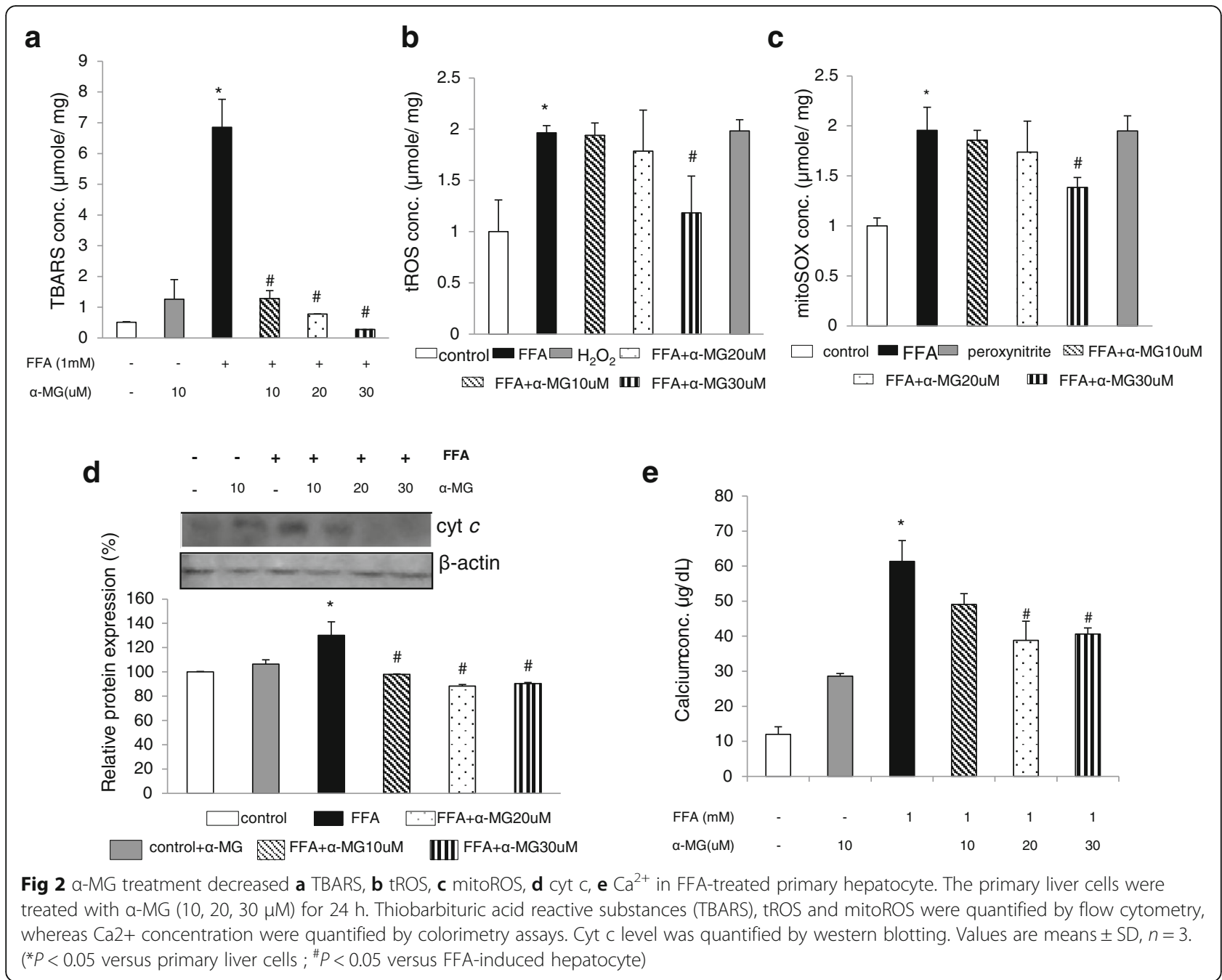

obese mice by activating SirT1-AMPK and PPARy pathways [12]. Overall, these data including in our study suggest that $\alpha-M G$ could potentially regulate hepatic steatosis in an obesity setting.

ROS are critical factors in the pathogenesis of NAFLD leading to cellular damage and mitochondrial dysfunction. As evident in our study, MGE enhanced the cellular antioxidant enzyme activities including GPx, GRd, SOD, CAT, and GSH (Table 1), which could explain the substantial decline in tROS (Fig. 2b), and mitoROS (Fig. 2c) in the MGE treatment. The reduced ROS levels implied increased cellular protection as supported by decline in TBARS (Fig. 2a), a potential indicator of oxidative cellular damage due to lipid peroxidation. Mangosteen pericarps are rich sources of xanthones bioactive compounds that could have enhanced the endogenous antioxidant defence. Diminished GSH has previously been implicated in the pathogenesis of alcohol liver diseases [18]. In a previous study, Marquez-Valadez et al. found $\alpha$-MG $(10 \mu \mathrm{M}, 25 \mu \mathrm{M}$ and $50 \mu \mathrm{M})$ significantly increased GPx activity in rat brain synaptosomes with 3NP-induced mitochondrial dysfunction [19]. Fang et al. studied ARPE-19 cells with $\mathrm{H}_{2} \mathrm{O}_{2}$-induced high cellular stress and found $\alpha-\mathrm{MG}$ treatment $(200 \mu \mathrm{M})$ decreased TBARS levels and increased SOD, GPx and GSH activities [20]. Shen et al. reported effects of $\alpha$-mangosteen in the regulation of antioxidant sensor NRF2 transcription factor on regulation of transcription and redox status in adipocyte [21]. The authors attributed these observations to $\alpha-M G$ regulation of mitogen-activated protein kinases (MAPKs), extracellular signal-regulated kinase (ERK)1/2, JNK and p38 protein expression [20].

Emerging evidence has shown defects in mitochondrial bioenergetics may have a role in the development of liver diseases especially in an obesity setting [18]. Mitochondrial dysfunction can lead to an imbalanced triglyceride accumulation via reduced mitochondrial fatty acids oxidation mechanism. Therefore, reduced ROS and suppressed TBARS in the $\alpha M G$ treatment suggested mitochondrial protection from oxidative damage. In our 

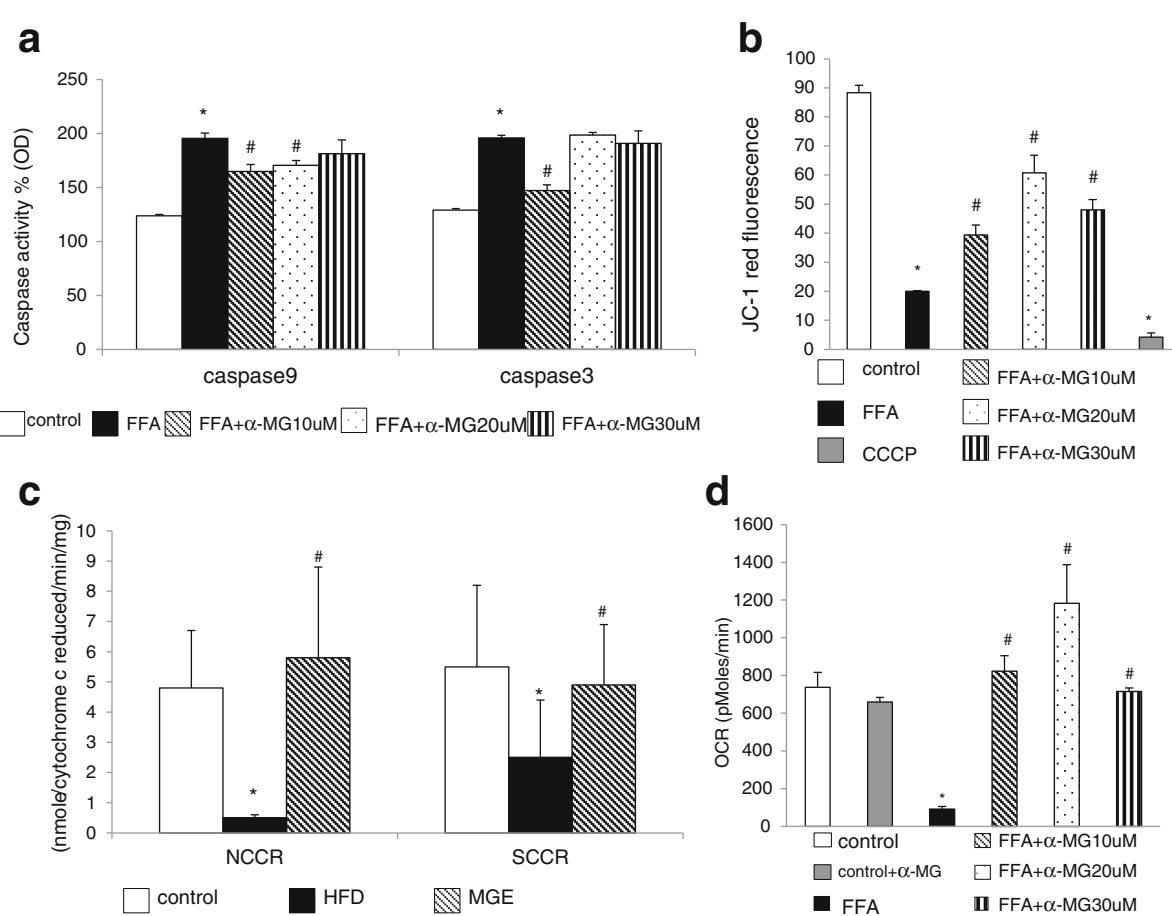

Fig 3 Effect of a-MG treatment on : a caspases 9 and 3 activities, $\mathbf{b}$ mitochondria membrane expressed as JC-1 fluorescence, $\mathbf{d}$ OXPHOS expressed as oxygen consumption rate in FFA-treated hepatocytes. The primary liver cells were treated with a-MG $(10,20,30 \mu \mathrm{M})$ for $24 \mathrm{~h}$. OXPHOS was quantified by seahorse XFe24 Analyzer. Caspases 9 and caspases 3 were quantified by Elisa assays. ${ }^{*} P<0.05$ versus primary liver cells; ${ }^{\#} P<0.05$ versus FFA-treated hepatocytes). Values are means $\pm S D, n=3$. Effect of mangosteen pericarp extract on mitochondrion complex enzymes (NCCR, SCCR) c. OXPHOS: oxidative phosphorylation. NCCR: NADH-cytochrome $c$ reductase. SCCR: succinate-cytochrome $c$ reductase. Values are mean \pm SD $(n=8) ;(* P<0.05$ versus control; ${ }^{\#} P<0.05$ versus HFD)

study, $\alpha$ MG treatment showed enhanced OCR (Fig. 3d), NCCR (mitochondrial complex I and III) and SCCR (mitochondrial complex II and III) (Fig. 3c), which are critical aspects of cell bioenergetics and mitochondrial oxidative phosphorylation relating to breakdown triglycerides for energy metabolism. In our study, improved mitochondrial oxidative phosphorylation is also evident from the enhanced mitochondrial membrane potential in the $\alpha M G$ treatment (Fig. $3 b$ ).

On the other hand, loss of mitochondrial membrane potential is strongly associated with apoptosis signalling via release of $\mathrm{Ca}^{2+}$, cytochrome $\mathrm{c}$ and caspases. As shown in our study, control group showed markedly reduced mitochondrial membrane (Fig. 3b), which corresponded to enhanced release of $\mathrm{Ca}^{2+}$ (Fig. 2e), cytochrome c (Fig. 2d), and caspase activity (Fig. 3a) compared to $\alpha \mathrm{MG}$ group. Our findings are comparable to those of Moravcova et al., who found $1 \mathrm{mM}$ fatty acid mix (palmitate: oleate acid, 2:1) treatment increased triglyceride, ROS, caspase 3 and reduced mitochondrial membrane potential $(\Delta \psi \mathrm{m})$ in primary liver cells, and further recommended in vitro models as suitable tools for studying hepatocellular consequences of steatosis [22]. Increased apoptosis of hepatocytes can lead to rapid cell death and inability of the cells to effectively modulate the lipid metabolism leading to hyperlipidemia. In our study, incorporation of $\alpha \mathrm{MG}$ improved mitochondrial membrane potential and suppressed levels of $\mathrm{Ca}^{2+}$, cytochrome $\mathrm{c}$, and caspase activity. Thus, the reduced triglyceride accumulation could partly be attributed to suppressed hepatocellular apoptosis via improved mitochondrial membrane potential and overall improved cell functions.

The alternative explanation for the reduced apoptosis levels in the MGE group can be due to the improved cellular antioxidant capacity as evident in the reduced tROS, increased antioxidant enzyme activities (Table 1), and reduced oxidative damage due to lipid peroxidation (Fig. 2a). ROS plays an important role in mediating apoptosis via redox signaling of ASKI signalosome/JNK pathways [9]. In an earlier study, Janhom and Dharmasaroja, suggested the inhibition effect of $\alpha-M G$ against $\mathrm{MPP}^{+}$-induced apoptosis in neuroblastoma SH-SY5Y cells may be associated with the reduction of ROS production, modulation of the balance of pro- and anti-apoptotic genes, and suppression of caspase-3 activation [23]. Kwak et al., in a recent study indicated $\alpha-M G$ treatment suppressed cell death via G1 phase arrest and downregulated cell cycle- 
related proteins $(\mathrm{CDK} / \mathrm{cyclin})$ in human oral squamous cell carcinoma [24]. Shan et al. reported $\alpha$-MG $(7 \mu \mathrm{g} / \mathrm{mL})$ treatment markedly suppressed human gastric adenocarcinoma cells via the constitutive Stat 3 protein activation, and Stat3-regulated Bcl-xL and Mcl-1 protein levels [25]. The question that remains in the current study is the potential molecular targets for the $\alpha-M G$ modulation of hepatic triglyceride accumulation and apoptosis, hence future studies are warranted to elucidate the molecular mechanisms by which $\alpha$-MG modulates hepatic lipid homeostasis via mitochondrial functionality and apoptosis pathways.

Overall, Fig. 4 describes our proposed model for the $\alpha$-MG regulatory effect on hepatic steatosis in high fat diet fed rats. High fat diet led to overload of FFA/ triglycerides, increased lipid peroxidation and ROS production in the liver, further suppressing the antioxidant capacity and causing mitochondrial damage. Mitochondrial damage led to increased cytochrome $c$ and $\mathrm{Ca}^{2+}$ release from mitochondria, and subsequent activation of caspases 9 and 3, eventually leading to hepatocyte apoptosis via mitochondria-dependent pathways. The mitochondrial damage in turn leads to a decrease in mitochondrial oxidative capacity, including $\beta$-oxidation and ATP production, and consequently excessive triglyceride accumulation in the liver. Upon supplementation of high fat diet with $\alpha-M G$ from mangosteen pericarps, the antioxidant enzymes activities were enhanced, leading to decreased ROS levels and increased hepatic mitochondrial oxidative phosphorylation capacity and efficient catabolism of accumulated hepatic triglyceride. On the other hand, $\alpha$-MG resulted in decreased hepatocyte apoptosis via improved mitochondrial integrity and diminished oxidative stress. This means that the liver cells role in lipid metabolism were not dysregulated by the programmed cell death arising from mitochondriadependent apoptosis. Chitchumroonchokcha et al. studied the bioavailability of xanthones in human subjects and observed all the participants completed the study with no gastrointestinal distress after consuming the test sample of $60 \mathrm{~mL}$ mangosteen juice $(59 \%$ $\alpha$-mangostin) [26]. The findings of this human study are a good indication on the safety of the $\alpha$-MG extract and the potential application of the $\alpha-M G$ in therapeutic management of NAFLD.

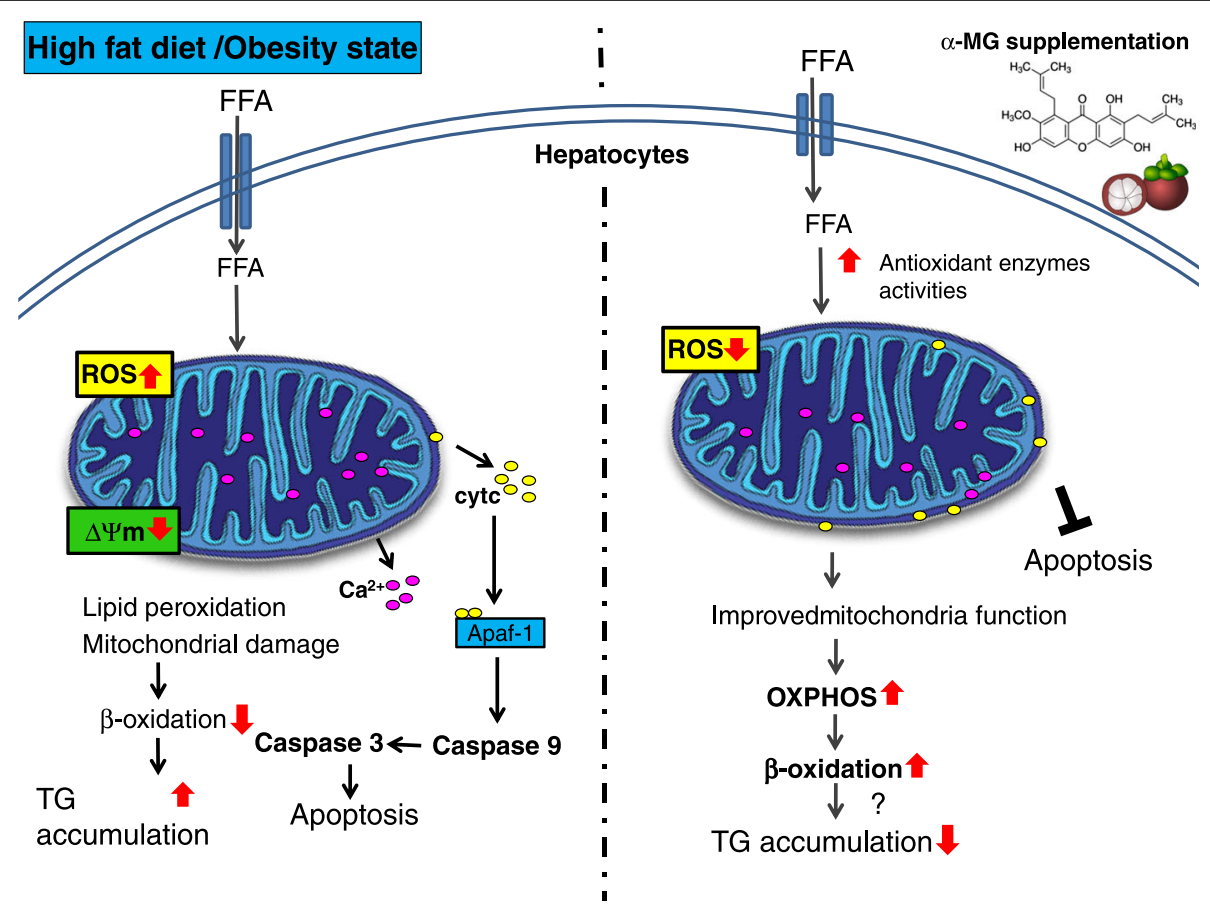

Fig. 4 Proposed mechanism for a-MG action against fat infiltration of the liver. High fat diet in an obesity setting, leads to overload of FFAs in hepatocytes. Consequently, there occurs increased ROS production and lipid peroxidation in the liver, which further damage the antioxidant enzymes and mitochondria. Mitochondrial damage leads to $c y t c$ and $\mathrm{Ca}^{2+}$ release from mitochondria, thereafter cyt $c$ binds to Apaf- 1 and activates apoptosis via caspases 9 and 3 factors. Apoptosis implies rapid death of hepatocytes hence reduced clearance of fat deposits. On the other hand, mitochondrial damage in turn leads to a decreased mitochondrial oxidative capacity, including $\beta$-oxidation and ATP production, resulting in TG accumulation in the liver. a-MG limits hepatic TG accumulation through enhanced anti-oxidative capacity and hepatic mitochondrial oxidative metabolic capacity and inhibition of mitochondrial pathway of apoptosis, which may confer decreased hepatic steatosis risk. ROS: reactive oxygen species; $\mathrm{Ca}^{2+}$ : calcium ; cyt c: cytochrome $c$; TG: triglyceride 


\section{Conclusion}

Our study demonstrates that $\alpha$-MG suppressed hepatic steatosis in high fat diet- fed rats via various mechanisms including increased antioxidant enzyme capacity, improved mitochondrial functionality and suppressed mitochondria-dependent apoptosis. These data indicate $\alpha-M G$ extracts from mangosteen pericarps may be used as a preventive agent for hepatosteatosis through regulating mitochondrial oxidative phosphorylation and apoptosis mechanisms.

\section{Abbreviations}

AMPK: Denosine 5'-monophosphate (AMP)-activated protein kinase; $\mathrm{Ca}^{2+}$ : Calcium; CAT: Catalase; CCCP: Carbonylcyanide p-trifluoromethoxyphenylhydrazone; cyt c. cytochrome $c$; ERK: Extracellular signal-regulated kinase; ETC: Electron transport chain; FBS: Fetal bovine serum; FFA: Free fatty acid; GPx: Glutathione peroxidase; GRd: Glutathione reductase; GSH: Glutathione; HFD: High-fat diet; MAPKs: Mitogen-activated protein kinases; MDA: Malondialdehyde MGE: Mangosteen pericarp extract; mitoROS: mitochondrial ROS; NAFLD: Nonalcoholic fatty liver disease; NCCR: NADH-cytochrome c reductase; SCCR: Succinate-cytochrome $c$ reductase; SD: Sprague Dawley; SOD: Superoxide dismutase; TBARS: Thiobarbituric acid-reactive substances; TG: Triglycerides; tROS: total reactive oxygen species; a-MG: a- mangostin; $\Delta \psi \mathrm{m}$ : Mitochondrial membrane potential

\section{Acknowledgement}

We thank Shinn Nan World Trade Co., Ltd. (Taipei, Taiwan) for providing mangosteen (Garcinia mangostana Linn.) pericarp extract.

\section{Funding}

This research work was funded by the authors from study design to publication.

\section{Availability of data and materials}

Data sharing not applicable to this article as no datasets were generated.

\section{Authors' contributions}

SHY participated in design and coordination of the study, collected data and participated in data interpretation, and helped to draft the manuscript. SHY participated in coordination of the cell study while PCC, IJT while PYW participated in coordination of the animal study. JIT participated in data collection and analysis. EO, TSY and RHH reviewed and approved the final manuscript. All authors read and approved the final manuscript.

\section{Competing interests}

The authors declare that they have no competing interests.

\section{Consent for publication}

Not applicable.

\section{Ethics approval and consent to participate}

The animal experiment protocol was reviewed and approved by the Institutional Animal Care and Use committee (IACUC/IACUP) at Taipei Medical University (IACUC approval No: LAC-2015-0109).

\section{Author details \\ 'School of Nutrition and Health Sciences, Taipei Medical University, 250 Wu-Hsing Street, Taipei 110, Taiwan. ${ }^{2}$ Institute of Food Bioresources and Technology, Dedan Kimathi University of Technology, P.O. Box 657-10100 Nyeri, Kenya. ${ }^{3}$ Yuan Lyu Technology Corporation, 10F-3, 120 Chung Cheng 1st Road, Kaohsiung 802, Taiwan. ${ }^{4}$ Institute of Anatomy and Cell Biology, School of Medicine, National Yang-Ming University, Taipei 112, Taiwan.}

Received: 25 July 2016 Accepted: 22 November 2016 Published online: 01 December 2016

\section{References}

1. Fabbrini E, Sullivan S, Klein S. Obesity and nonalcoholic fatty liver disease: biochemical, metabolic, and clinical implications. Hepatology. 2010;51:679-89.

2. Dongiovanni $P$, Lanti C, Riso P, Valenti L. Nutritional therapy for nonalcoholic fatty liver disease. J Nutr Biochem. 2016:29:1-11.

3. Cohen JC, Horton JD, Hobbs HH. Human fatty liver disease: old questions and new insights. Science. 2011;332:1519-23.

4. Chae HS, Kim YM, Bae JK, Sorchhann S, Yim S, Han L, Paik JH, Choi YH, Chin YW. Mangosteen Extract Attenuates the Metabolic Disorders of High-FatFed Mice by Activating AMPK. J Med Food. 2016;19:148-54.

5. Marchi S, Giorgi C, Suski JM, Agnoletto C, Bononi A, Bonora M, De Marchi E, Missiroli S, Patergnani S, Poletti F, et al. Mitochondria-ros crosstalk in the control of cell death and aging. J Signal Transduct. 2012;2012:329635.

6. Sreekumar R, Unnikrishnan J, Fu A, Nygren J, Short KR, Schimke J, Barazzoni $R$, Nair KS. Impact of high-fat diet and antioxidant supplement on mitochondrial functions and gene transcripts in rat muscle. Am J Physiol Endocrinol Metab. 2002;282:E1055-61

7. Han CW, Kang ES, Ham SA, Woo HJ, Lee JH, Seo HG. Antioxidative effects of Alisma orientale extract in palmitate-induced cellular injury. Pharm Biol. 2012;50:1281-8

8. Wang K. Molecular mechanisms of hepatic apoptosis. Cell Death Dis. 2014;5:e996.

9. Sinha K, Das J, Pal PB, Sil PC. Oxidative stress: the mitochondria-dependent and mitochondria-independent pathways of apoptosis. Arch Toxicol. 2013;87:1157-80.

10. Chin YW, Kinghorn AD. Structural Characterization, Biological Effects, and Synthetic Studies on Xanthones from Mangosteen (Garcinia mangostana), a Popular Botanical Dietary Supplement. Mini Rev Org Chem. 2008;5:355-64.

11. Devi Sampath P, Vijayaraghavan K. Cardioprotective effect of alphamangostin, a xanthone derivative from mangosteen on tissue defense system against isoproterenol-induced myocardial infarction in rats. J Biochem Mol Toxicol. 2007;21:336-9.

12. Choi YH, Bae JK, Chae HS, Kim YM, Sreymom Y, Han L, Jang HY, Chin YW. Alpha-Mangostin Regulates Hepatic Steatosis and Obesity through SirT1AMPK and PPARgamma Pathways in High-Fat Diet-Induced Obese Mice. J Agric Food Chem. 2015;63:8399-406.

13. Seglen PO. Preparation of isolated rat liver cells. Methods Cell Biol. 1976;13:29-83.

14. Kim CS, Kwon Y, Choe SY, Hong SM, Yoo H, Goto T, Kawada T, Choi HS, Joe Y, Chung HT, Yu R. Quercetin reduces obesity-induced hepatosteatosis by enhancing mitochondrial oxidative metabolism via heme oxygenase-1. Nutr Metab (Lond). 2015;12:33.

15. Taher M, Mohamed Amiroudine MZ, Tengku Zakaria TM, Susanti D, Ichwan SJ, Kaderi MA, Ahmed QU, Zakaria ZA. Alpha-Mangostin Improves Glucose Uptake and Inhibits Adipocytes Differentiation in 3 T3-L1 Cells via PPARgamma, GLUT4, and Leptin Expressions. Evid Based Complement Alternat Med. 2015;2015:740238.

16. Folch J, Lees M, Sloane Stanley GH. A simple method for the isolation and purification of total lipides from animal tissues. J Biol Chem. 1957:226:497-509.

17. Park S-Y, Bok S-H, Jeon S-M, Park YB, Lee S-J, Jeong T-S, Choi M-S. Effect of rutin and tannic acid supplements on cholesterol metabolism in rats. Nutr Res. 2002;22:283-95.

18. Mantena SK, King AL, Andringa KK, Eccleston HB, Bailey SM. Mitochondrial dysfunction and oxidative stress in the pathogenesis of alcohol- and obesity-induced fatty liver diseases. Free Radic Biol Med. 2008:44:1259-72.

19. Marquez-Valadez B, Maldonado PD, Galvan-Arzate S, Mendez-Cuesta LA, Perez-De La Cruz V, Pedraza-Chaverri J, Chanez-Cardenas ME, Santamaria A. Alpha-mangostin induces changes in glutathione levels associated with glutathione peroxidase activity in rat brain synaptosomes. Nutr Neurosci. 2012;15:13-9.

20. Fang $Y$, Su T, Qiu X, Mao P, Xu Y, Hu Z, Zhang Y, Zheng X, Xie P, Liu Q. Protective effect of alpha-mangostin against oxidative stress induced-retinal cell death. Sci Rep. 2016;6:21018.

21. Shen Q, Chitchumroonchokchai C, Thomas JL, Gushchina LV, Disilvestro D, Failla ML, Ziouzenkova O. Adipocyte reporter assays: application for identification of anti-inflammatory and antioxidant properties of mangosteen xanthones. Mol Nutr Food Res. 2014;58:239-47.

22. Moravcova A, Cervinkova Z, Kucera O, Mezera V Rychtrmoc D, Lotkova H. The effect of oleic and palmitic acid on induction of steatosis and 
cytotoxicity on rat hepatocytes in primary culture. Physiol Res. 2016;64 Suppl 5:S627-36.

23. Janhom P, Dharmasaroja P. Neuroprotective Effects of Alpha-Mangostin on MPP(+)-Induced Apoptotic Cell Death in Neuroblastoma SH-SY5Y Cells. J Toxicol. 2015;2015:919058.

24. Kwak HH, Kim IR, Kim HJ, Park BS, Yu SB. Alpha-Mangostin Induces Apoptosis and Cell Cycle Arrest in Oral Squamous Cell Carcinoma Cell. Evid Based Complement Alternat Med. 2016;2016:5352412.

25. Shan T, Cui XJ, Li W, Lin WR, Lu HW, Li YM, Chen X, Wu T. Alpha-Mangostin suppresses human gastric adenocarcinoma cells in vitro via blockade of Stat3 signaling pathway. Acta Pharmacol Sin. 2014;35:1065-73.

26. Chitchumroonchokchai C, Riedl KM, Suksumrarn S, Clinton SK, Kinghorn AD, Failla ML. Xanthones in mangosteen juice are absorbed and partially conjugated by healthy adults. J Nutr. 2012;142:675-80.

Submit your next manuscript to BioMed Central and we will help you at every step:

- We accept pre-submission inquiries

- Our selector tool helps you to find the most relevant journal

- We provide round the clock customer support

- Convenient online submission

- Thorough peer review

- Inclusion in PubMed and all major indexing services

- Maximum visibility for your research

Submit your manuscript at www.biomedcentral.com/submit
Biomed Central 